\title{
A rare case of back pain: sapho syndrome
}

\begin{abstract}
Back pain is one of the most common reasons for outpatient visits but is rarely severe enough to require hospitalization. Synovitis-acne-pustulosis-hyperostosis-osteitis (SAPHO) syndrome, a chronic inflammatory disorder is one of the rare causes of back pain which occurs in combination with skin symptoms. Here, we present a case of SAPHO syndrome in a 39-year-old man who presented with osteoarticular and dermatologic manifestations.
\end{abstract}

Keywords: SAPHO syndrome, back pain, osteitis, hypertension, elevated sedimentation rate, computed tomography, C-reactive protein

\author{
Volume I4 Issue 3 - 202 I \\ Roopali Goyal Gandhi,' Leela Krishna Teja \\ Boppana,' Sravani Lokineni,' Megha Garg² \\ 'Department of Internal Medicine, Rochester General Hospital, \\ USA \\ ${ }^{2}$ Department of Allergy, Immunology, \& Rheumatology, \\ Rochester General Hospital, USA
}

\author{
Correspondence: Sravani Lokineni, Department of Internal \\ Medicine, Rochester General Hospital, 1425 Portland Avenue, \\ Rochester, NY, I462I, USA, \\ Email sravani.lokineni@rochesterregional.org
}

Received: May 20, 202I | Published: June 24, 2021
Abbreviations: CRP, C-reactive protein; CT, computed tomography; ESR, elevated sedimentation rate; MRI, magnetic resonance imaging

\section{Case presentation}

A 39-year-old man with a medical history of hypertension and chronic back pain presented to the hospital with complaints of acute on chronic mid-thoracic back pain without preceding fall or trauma. There was no lower extremity weakness or urinary or fecal incontinence, but he did report fevers. There was also a new rash, which had started on his heel and palms the day before presentation. There was no history of intravenous drug use, and he reported severe cystic acne as a teenager. On physical examination, he was noted to be febrile $\left(100.8^{\circ} \mathrm{F}\right)$, with tenderness elicited over the thoracic spine and with pustular lesions on the palms (Figure 1A) and the medial aspect of the right heel. Laboratory investigations showed leukocytosis of $16900 / \mu 1$, an elevated sedimentation rate $(\mathrm{ESR})$ of $103 \mathrm{~mm} / \mathrm{hr}$, and
C-reactive protein (CRP) of $319 \mathrm{mg} / \mathrm{ml}$. Computed tomography (CT) of thoracic spine showed paraspinal soft tissue thickening from T4T10 with vertebral body sclerosis, endplate degenerative changes and bridging syndesmophytes. Magnetic resonance imaging (MRI) of the thoracic spine showed diffuse moderate disc space narrowing from T4-5 through T10-11, consistent with degenerative changes, and endplate signal abnormalities at T10-11 with discogenic marrow changes of the T10 and T11 vertebrae (Figure 1B). These imaging findings were highly suspicious for discitis and patient was treated with broad spectrum antibiotics but the blood cultures and bone biopsy turned out to be negative for infection. Rheumatology was consulted, and SAPHO syndrome was diagnosed, given that we had excluded an infectious etiology and given the skin manifestations of palmoplantar pustulosis, hyperostosis, and osteitis on imaging which was initially thought as discitis. He was started on steroid taper and NSAIDs and was discharged home. On follow-up, he was started on adalimumab (Humira) given the spine involvement with significant improvement in his back pain and also skin lesions.
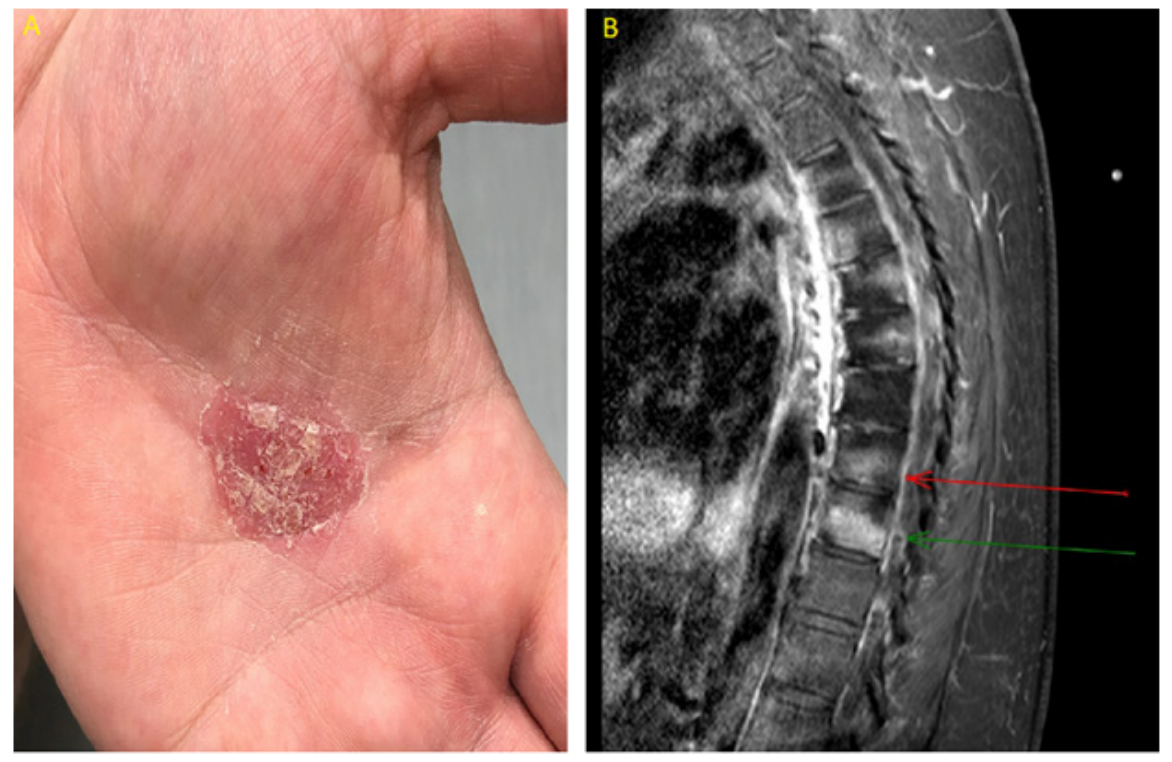

Figure I (A) Pustular skin lesion on the palmar aspect (B) Sagittal TI weighted post contrast MRI thoracic spine showing the enhancing areas (red and green arrows) of the lower thoracic bodies suggestive of active inflammation or osteitis. 


\section{Discussion}

SAPHO syndrome is a chronic inflammatory disorder manifesting with osteoarticular and dermatologic involvement and a relapsingremitting disease course. It was first described in 1987 by Chamot et al. ${ }^{1}$ and there have since been many case reports in the literature, yet it is still considered a rare disease whose exact prevalence is unknown. It affects patients across all age groups, and its etiology is thought to be multifactorial, involving genetic, infectious, and immunological components. The diagnosis, which our patient met, is based on the exclusion of infectious arthritis or osteomyelitis and the presence of one of four diagnostic criteria, ${ }^{1}$ proposed by Benhamou ${ }^{2}$ in 1988: skin manifestations of severe acne; skin manifestations of palmoplantar pustulosis; hyperostosis, with or without dermatosis; and chronic recurrent multifocal osteomyelitis involving axial or peripheral skeleton, with or without dermatosis. Laboratory investigations are non-specific and can show elevated inflammatory markers ESR and CRP, but radiologic investigation ${ }^{3}$ with CT, MRI, and bone scintigraphy can be particularly helpful in early diagnosis. The notable imaging ${ }^{3}$ findings include cortical erosion, sclerosis, and paravertebral bony bridging on CT and osteitis and hyperostosis on MRI. Simple X-ray ${ }^{3}$ can be normal for about three months after disease onset in about $80 \%$ of patients. Patients usually have polyostotic ${ }^{3}$ involvement, with the spine being the second most commonly involved site after the chest wall. In a study ${ }^{4}$ that compared the clinical and imaging features of SAPHO syndrome, patients with spinal involvement were found to suffer a longer disease and have more intense inflammatory reactions. Treatment ${ }^{5}$ primarily involves NSAIDs; disease-modifying antirheumatic agents, like methotrexate, sulfasalazine, and leflunomide, which are particularly useful in peripheral arthritis and; agents like tumor necrosis factor inhibitors ${ }^{5}$ (e.g., infliximab, adalimumab, etanercept), have been found to be helpful in axial arthritis. Oral retinoids and antibiotic treatment with tetracycline have been helpful in the treatment of acne and extensive palmoplantar pustulosis.

\section{Conclusion}

SAPHO syndrome is an under-recognized disease. The heterogeneity of the clinical and radiological manifestations can lead to delayed diagnosis. Early treatment can result in decreased disease progression and improved quality of life.

\section{Acknowledgments}

None.

\section{Conflicts of interest}

The rest of the authors declare do not have conflicts of interest.

\section{Funding}

This manuscript was not supported by any funding.

\section{References}

1. Chamot AM, Benhamou CL, Kahn MF, et al. Le syndrome acné pustulose hyperostose ostéite (SAPHO). Résultats d'une enquête nationale. 85 observations [Acne-pustulosis-hyperostosis-osteitis syndrome. Results of a national survey. 85 cases]. Rev Rhum Mal Osteoartic. 1987;54(3):187-196.

2. Benhamou CL, Chamot AM, Kahn MF. Synovitis-acne-pustulosis hyperostosis-osteomyelitis syndrome (SAPHO). A new syndrome among the spondyloarthropathies? Clin Exp Rheumatol. 1988;6(2):109-112.

3. Himuro $\mathrm{H}$, Kurata $\mathrm{S}$, Nagata $\mathrm{S}$, et al. Imaging features in patients with SAPHO/CRMO: a pictorial review. Jpn J Radiol. 2020;38(7):622-629.

4. Gao S, Deng X, Zhang L, et al. The comparison analysis of clinical and radiological features in SAPHO syndrome. Clin Rheumatol. 2021;40(1):349-357.

5. Liu S, Tang M, Cao Y, et al. Synovitis, acne, pustulosis, hyperostosis, and osteitis syndrome: review and update. Ther Adv Musculoskelet Dis. 2020;12:1759720X20912865. 